\title{
Diffusion of cosmic rays in heliosphere, observations from GRAPES-3
}

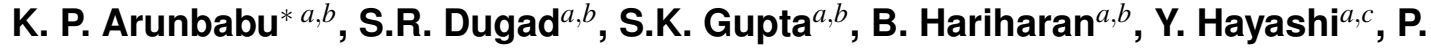 \\ Jagadeesan $^{a, b}$, A. Jain ${ }^{a, b}$, S. Kawakami ${ }^{a, c}$, H. Kojima ${ }^{a, d}$, P.K. Mohanty ${ }^{a, b}$, S.D. \\ Morris $^{a, b}$, P.K. Nayak ${ }^{a, b}$, A. Oshima ${ }^{a, e}$, B.S. Rao ${ }^{a, b}$, S. Shibata ${ }^{a, e}$, P. Subramanian ${ }^{a, f}$ \\ ${ }^{a}$ GRAPES-3 Experiment, Cosmic Ray Laboratory, Raj Bhavan, Ooty 643001, India \\ ${ }^{b}$ Tata Institute of Fundamental Research, Mumbai 400005, India \\ ${ }^{c}$ Graduate School of Science, Osaka City University, Osaka 558-8585, Japan \\ ${ }^{d}$ Faculty of Engineering, Aichi Insitute of Technology, Toyota City, Aichi 470-0392, Japan \\ ${ }^{e}$ College of Engineering, Chubu University, Kasugai, Aichi 487-8501, Japan \\ ${ }^{f}$ Indian Institute of Science Education and Research, Pune, 411008, India \\ E-mail: arun.babu@tifr.res.in
}

The large area $\left(560 \mathrm{~m}^{2}\right)$ GRAPES-3 tracking muon telescope has been operating at Ooty, India uninterruptedly from 2001. It records $4 \times 10^{9}$ muons of energy $>1 \mathrm{GeV}$ with an angular resolution of $\sim 4^{\circ}$ every day. The high statistical accuracy of this instrument helps in measuring the variations of galactic cosmic rays (GCRs) with a statistical precision of $<0.01 \%$ every hour. Here a study of solar modulation of GCR using GRAPES- 3 is presented. We analysed uninterrupted GRAPES-3 data for six years (2005-2010) after applying the detector efficiency, pressure, and temperature corrections on this data to remove the effects of instrumental and atmospheric variations. Muon intensity, after applying these corrections shows an anti-correlation with the interplanetary magnetic field (IMF) from ACE/WIND spacecrafts. This anti-correlation of muon intensity with the IMF indicates that the solar modulations of GCRs may be caused by variations in the IMF. The dependence of GCR intensity variation and IMF is estimated. Further calculations were performed to study these variations by assuming the diffusion of GCRs through the turbulent magnetic field. The results show that the solar modulations of GCRs are largely due to the diffusion of GCRs though turbulent IMF in the heliosphere.

Keywords: cosmic rays, interplanetary magnetic field (IMF), solar wind, diffusion.

35th International Cosmic Ray Conference - ICRC2017

10-20 July, 2017

Bexco, Busan, Korea

*Speaker. 


\section{Introduction}

The modulation of galactic cosmic ray (GCR) intensity by the solar wind has been a subject of considerable interest for decades. The heliosphere is vast, which extends to $\sim 120$ AU. The GCRs encounter a turbulent solar wind with an embedded magnetic field when entering the heliosphere, which leads to significant global and temporal variations in their intensity and in their energy as a function of position inside the heliosphere. Understanding the behaviors of GCRs with time over the solar cycle is a way to understand the behaviors of heliosphere as a whole. The temporal variation of the GCR intensity in the heliosphere is known as cosmic-ray modulation. The main periodicity that are of solar origin are 11-year solar activity cycle, 27-day solar rotation variation, a diurnal variation, and irregular variations such as Forbush decreases [1, 2, 3, 4, 5, 6, 7].

Diffusion of GCRs through the turbulent magnetic field is also a topic of great interest $[8,9]$. The magnetic field dependence and GCR rigidity dependence on the observed GCR flux is an important factor in the formulation of diffusion approximation of GCRs through the turbulent magnetic field. In this study we are investigating the magnetic field dependence of the observed GCR flux using the GRAPES-3 tracking muon telescope, and investigating how the different diffusion approximations can be used to explain these observations.

\section{The GRAPES-3 experiment}

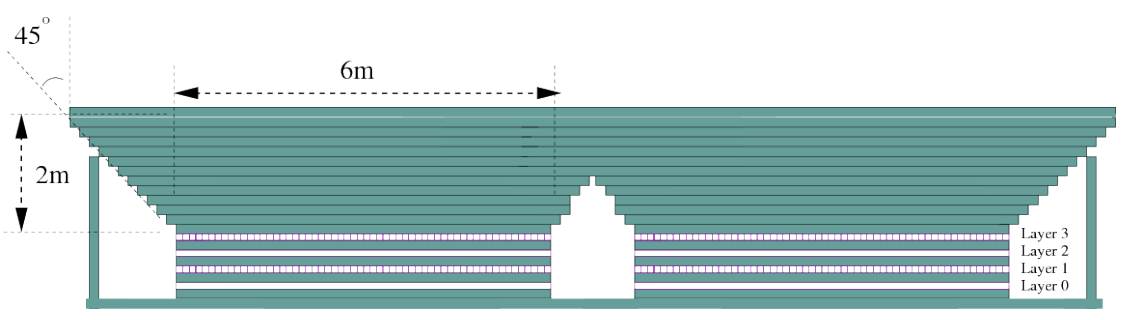

Figure 1: Schematic of a muon detector supermodule with 4 layers of proportional counters (PRCs) embedded in concrete blocks.

The GRAPES-3 experiment is located at Ooty at $11.4^{\circ} \mathrm{N}$ latitude, $76.7^{\circ} \mathrm{E}$ longitude, and $2200 \mathrm{~m}$ altitude in India. There are two main components of GRAPES-3 experiment. The first one is an extensive air shower (EAS) array of 400 plastic scintillator detectors, each of them have an area of $1 \mathrm{~m}^{2}$, which are placed on a hexagonal pattern with a minimum separation of $8 \mathrm{~m}$. This EAS array is used to trigger on showers of energy $\geq 10 \mathrm{TeV}$ [10]. A large area $\left(560 \mathrm{~m}^{2}\right)$ tracking muon telescope constitutes the second component of GRAPES-3 experiment. The muon telescope comprises of sixteen modules each of them have $35 \mathrm{~m}^{2}$ area. This is an ideal instrument to study various phenomena at high energies caused by solar activities such the flares, coronal holes, CMEs etc. $[1,2,3,7,11,12,13]$. Complementary information on an EAS provided by these two independent components has made sensitive measurements of the composition and energy spectrum of primary cosmic rays in the energy range $10^{13}-10^{16} \mathrm{eV}$ possible $[14,15]$.

The basic element in a muon telescope module is the proportional counter (PRC), which were made from a square cross-section steel tube with dimensions of $600 \mathrm{~cm} \times 10 \mathrm{~cm} \times 10 \mathrm{~cm}$, and a 
wall thickness of $2.3 \mathrm{~mm}$. Muon modules are assemblies of PRCs placed in a four-layer configuration with consecutive layers arranged in mutually perpendicular directions as may be seen from Fig. 1. Four adjacent modules located under common concrete shielding constitute a supermodule. The cross-section of the two of these modules is visible in Fig. 1. An overburden of $550 \mathrm{~g} \mathrm{~cm}^{-2}$ in form of concrete blocks above the bottom most PRC layer was responsible for a threshold energy of $\sec (\theta) \mathrm{GeV}$ for the muons in a direction of zenith angle ' $\theta$ '. Each telescope module was instrumented to continuously record the intensity of muons in a matrix of $13 \times 13$ directions which cover a solid angle of $2.3 \mathrm{sr}$, once every $10 \mathrm{~s}$.

\section{Data analysis}

Each module in GRAPES-3 tracking muon telescope detects muons at a rate of $\sim 3000 \mathrm{~s}^{-1}$, therefore, for the full telescope, total recorded rate of muons was $3 \times 10^{6} \mathrm{~min}^{-1}$. This high-statistics, directional record of the muon intensity allows to identify the even very small changes in muon rate. In this analysis we used the 3 hour average muon rate for a period of six years from 20052010 , this averaging resulted in a typical statistical error of $<0.01 \%$. During the six-year interval (2005-2010), the efficiency of the sixteen modules underwent gradual changes. For precision studies it was necessary to correct for these slow changes in the efficiency during this interval. This was successfully done by exploiting the slow, and small changes in the efficiency of each module which were independent, and distinct from the remaining modules [16]. Following the correction of efficiency for the sixteen modules for the six-year interval, the measured muon intensities from these modules agreed within the statistical accuracy of the data $[17,18]$.

After correcting for the changes in the efficiency of each module for full six years, appropriate corrections were made for atmospheric modulations. Atmospheric pressure at Ooty shows a 12 hour periodic nature, the muon rate observed at Ooty shows a dependence on atmospheric pressure, which is anti correlated with the pressure. Since The solar diurnal variations also present along with the pressure modulations we used a the fast Fourier transform (FFT) method to find the pressure coefficient and performed the pressure correction [7, 19].

Muon intensity observed at Earth also shows a dependence on temperature of higher atmosphere. GRAPES-3 detect low-energy (vertical direction median rigidity $77 \mathrm{GeV}$ ) muons. The mesons responsible for the production of them were decayed in top of the atmosphere. An increase in the atmospheric temperature causes an expansion, thereby increasing the probability of the decay of the muons produced resulting in resulting in a reduction of muon intensity [20]. The daily variation in temperature of the upper atmosphere is negligible compared with the seasonal variations. Muon intensity observed in GRAPES-3 shows and anti-correlations with the effective temperature of upper atmosphere. Since the solar modulations of cosmic rays are present along with the seasonal variations, we used the FFT method to calculate the temperature coefficient and an appropriate temperature correction were performed to remove the seasonal variation from the data [21].

During this six years we have seen Forbush decreases up to a magnitude of 3\%, Forbush decreases are in good anti-correlation with the magnetic field compression associated with the CME engulfing Earth with a lag [2]. For this study we are interested in slower scale variation of GCRs with IMF we used a 60 day running average filter to remove the sudden variations of GCR 
and IMF, This will remove the large FD variations and the corresponding IMF compressions. These data are shown in figure 2. The GCR variations observed in GRAPES-3 for the six years (20052010) after applying the atmospheric corrections are shown in the first panel of figure ??. The IMF $\left(B_{\text {scalar }}\right)$ observed by the spacecrafts (ACE/WIND) during this period is shown in the second panel of figure 2 .

From the figure 2, we can see that the GCR variation depends up on the IMF variation. The dependence of GCR intensity on IMF ( $\left.B_{\text {scalar }}\right)$ is shown in figure 3 as black circles. Each black point in figure 3 represents the mean percent deviation of the muon intensity within a IMF range of $0.1 n T$ which is also displayed as a horizontal error bar. The vertical error bar at each point represents the root mean square spread in the muon intensity.

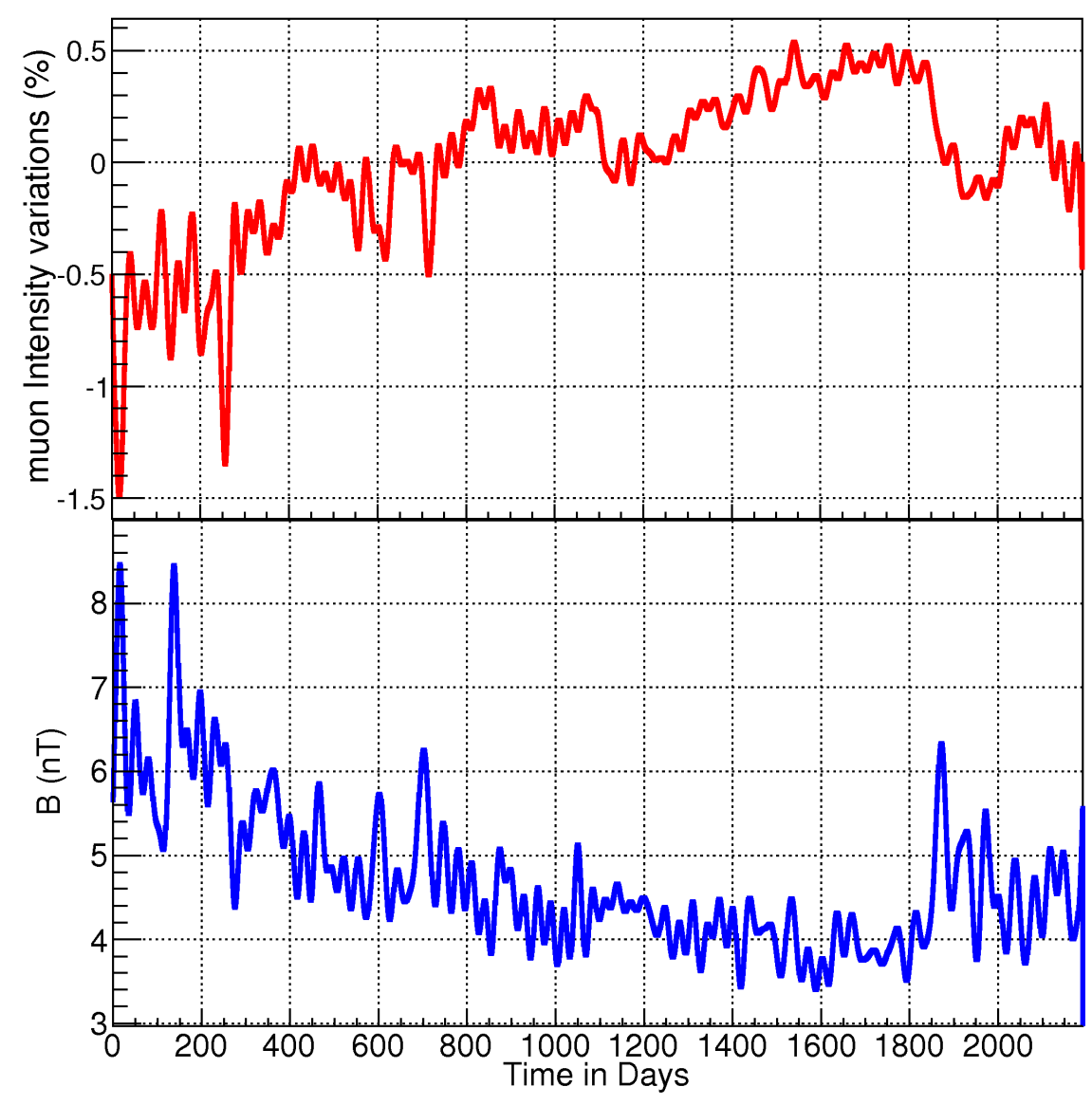

Figure 2: The first panel shows the CR intensity after applying the atmospheric corrections, second panel shows the IMF $B_{\text {scalar }}$

\section{Diffusion through heliosphere}

Our heliosphere is filled with the solar wind plasma, the interplanetary magnetic field associated with this plasma in the heliosphere can be considered as a magnetic spheroid, and GCRs are diffusion in to this magnetic shell. As we see a variation in the magnetic field of this shell we can see a corresponding variation in the GCR intensity. 
The flux of GCR protons diffusing into our heliosphere at a given time is given by

$$
F \quad=\quad D_{\perp} \frac{d N_{a}}{d r}
$$

where, $D_{\perp}$ is the perpendicular diffusion coefficient and $\frac{d N_{a}}{d r}$ is the density gradient of GCR.

We assumed a shell of magnetic plasma with the Sun at center and the heliopause as boundary, magnetic field varies in this shell radially outward inversely proportional to distance. If we consider the flux of protons measured at Earth, ie at $1 \mathrm{AU}$, it is given by the total number of cosmic rays diffusing upto $1 \mathrm{AU}$ from the boundary of the magnetic shell, and it can be represented as

$$
U=\int_{R_{h}}^{1 A U} A(r) F(r) d r . \quad=\quad \int_{R_{h}}^{1 A U} A(r) D_{\perp}(b, r) \frac{d N_{a}}{d r} d r .
$$

where $A(r)$ is the area of the part of spheroid of the shell at a distance ' $r$ ', which is proportional to $r^{2}$. The GCR density gradients can be expressed as

$$
\frac{d N_{a}}{d r}=\frac{N_{a}}{L}=\frac{N_{a}}{\kappa R_{L}(b)^{x}}
$$

where density gradient length scale $\mathrm{L}=\kappa R_{L}(b)^{x}, \kappa$ is proportionality constant and $R_{L}$ is Larmor radius, $\mathrm{x}$ is the power index.

Assuming the area $\mathrm{A}(\mathrm{r})=\mathrm{a} r^{2}$, and using the expression for density gradient we get total number of cosmic rays diffusing as,

$$
U=\int_{R_{h}}^{1 A U} a r^{2} D_{\perp}(b, r) \frac{N_{a}}{\kappa R_{L}(b)^{x}} d r .
$$

Percentage deviation of CR intensity calculated using deviation of CR intensity from mean over 6 year intensity, which is corresponds to a magnetic field $\sim 4.69 n T$, which here after be called as 'bm' in following calculations.

We use the same formalism as given in equation 4.4 and calculate the percentage deviation of GCR intensity (P.D) as ,

$$
P . D=100 \times \frac{U(b)-U(b m)}{U(b m)}=100 \times\left(\frac{U(b)}{U(b m)}-1\right)
$$

which can be expressed as

$$
\begin{gathered}
P . D=100 \times\left(\frac{\int_{R_{h}}^{1 A U} a r^{2} D_{\perp}(b, r) \frac{N_{a}}{\kappa R_{L}(b)^{x}} d r .}{\int_{R_{h}}^{1 A U} a r^{2} D_{\perp}(b m, r) \frac{N_{a}}{\kappa R_{L}(b m)^{x}} d r .}-1\right) \\
P . D=100 \times\left(\frac{\int_{R_{h}}^{1 A U} \frac{r^{2} D_{\perp}(b, r)}{R_{L}(b)^{x}} d r .}{\int_{R_{h}}^{1 A U} \frac{r^{2} D_{\perp}(b m, r)}{R_{L}(b m)^{x}} d r .}-1\right)
\end{gathered}
$$

In our further calculation we used two different expressions for the $D_{\perp}$, and the free parameter used for fitting the data was the power index ' $x$ ' 


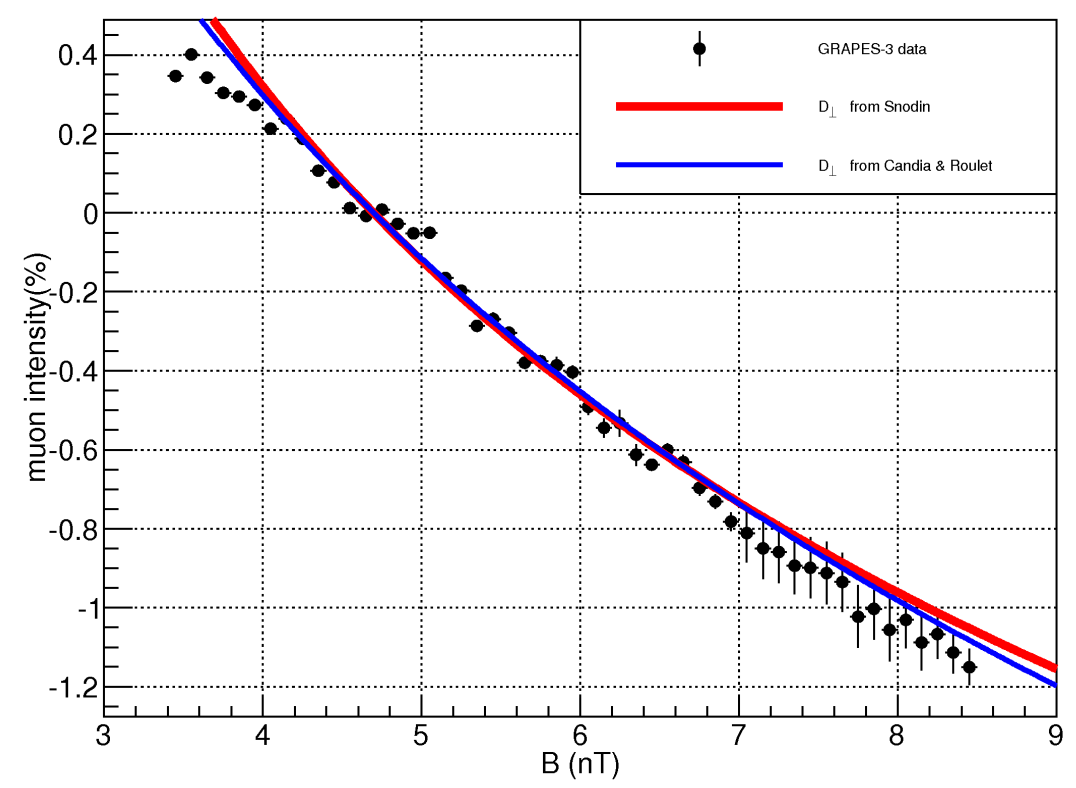

Figure 3: CR intensity versus $B_{\text {scalar }}$, red dots are GRAPES-3 data and the blue line is the model prediction using $D_{\perp}$ from Snodin et. al.

\section{1 $D_{\perp}$ from Candia \& Roulet(2004)}

The $\mathrm{D}_{\perp}$ prescription we used here is given by Candia \& Roulet (2004) [8], obtained from extensive Monte Carlo simulations of cosmic rays propagating through tangled magnetic fields.

Candia \& Roulet (2004) give the following fit for the 'parallel' diffusion coefficient $\mathrm{D}_{\|}$

$$
D_{\|} \equiv c L_{\max } \rho \frac{N_{\|}}{\sigma^{2}} \sqrt{\left(\frac{\rho}{\rho_{\|}}\right)^{2(1-\gamma)}+\left(\frac{\rho}{\rho_{\|}}\right)^{2}}
$$

where $\mathrm{c}$ is the speed of light and the quantities $\mathrm{N}_{\|}, \gamma$, and $\rho_{\|}$are constants specific to different kinds of turbulence whose values are listed in Table 1 of Candia \& Roulet (2004). $\mathrm{L}_{\max }$ is the maximum length scale of turbulence, in our case we considered it as 1 AU. The quantity $\rho$ is related to the rigidity of the proton $\mathrm{Rg}$ as,

$$
\rho=\frac{\mathrm{R}_{\mathrm{L}}}{\mathrm{L}_{\max }}=\frac{\mathrm{Rg}}{\mathrm{B}_{0} \mathrm{~L}_{\max }}
$$

where $\mathrm{R}_{\mathrm{L}}$ is the Larmor radius and $\mathrm{B}_{0}$ is the magnetic field. The quantity $\sigma^{2}=\left(\frac{\left\langle B_{t u r}^{2}\right\rangle}{B_{0}^{2}}\right)$ is the magnetic turbulence level, In this calculation we assumed a turbulence level $\sigma=10 \%$, which is an mean approximation of the value of $\sigma$ for the quiescent solar wind that ranges from 6 to $15 \%$ [22].

The perpendicular diffusion coefficient $\left(D_{\perp}\right)$ is related to the parallel one $\left(D_{\|}\right)$by,

$$
\frac{\mathrm{D}_{\perp}}{\mathrm{D}_{\|}} \equiv \begin{cases}N_{\perp}\left(\sigma^{2}\right)^{a_{\perp}}, & (\rho \leq 0.2) \\ N_{\perp}\left(\sigma^{2}\right)^{a_{\perp}}\left(\frac{\rho}{0.2}\right)^{-2}, & (\rho \geq 0.2)\end{cases}
$$


We used a Chi-square minimisation to get the value of free parameter i. e., the power index ' $\mathrm{x}$ ' used in the expression of the density gradient in equation 4.3 and thereafter.The resultant model prediction of CR intensity variation with the power index value $\mathrm{x}=0.3148$ for different magnetic field is given in the figure 3 in the blue line.

\section{2 $D_{\perp}$ from Snodin et al (2016)}

We used the formalism for $D_{\perp}$ given in Snodin et al (2016) [9], which was obtained by test particle simulations in random magnetic fields. The perpendicular diffusion coefficient, $D_{\perp}$ is given by

$$
\frac{D_{\perp}(b, \eta)}{c L_{m}}=\frac{\eta \frac{k_{0}(b)}{c L_{m}}+0.19(1-\eta)\left(\frac{R_{L}(b)}{L_{m}}\right)^{0.61}}{1+\left(\frac{1-\eta}{\eta}\right) \chi}
$$

here the quantity $\eta$ is equivalent the magnetic turbulence level, in this calculation we assumed a turbulence level $\sigma=10 \%$, which is an mean approximation of the value of $\sigma$ for the quiescent solar wind that ranges from 6 to $15 \%$ [22], and $L_{m}$ is considered as 1 AU. The value of $\chi=2.44$, and ' $c$ ' is the speed of light. The term $\frac{k_{0}(b)}{c L_{m}}$ is given by,

$$
\frac{k_{0}(b)}{c L_{m}}=0.0031+0.74 \frac{R_{L}(b)}{L_{m}}
$$

We used a Chi-square minimisation to get the value of free parameter i. e., the power index ' $\mathrm{x}$ ' used in the expression of the density gradient in equation 4.3 and thereafter.The resultant model prediction of CR intensity variation with the power index value $\mathrm{x}=0.6047$ for different magnetic field is given in the figure 3 in the red thick line.

\section{Discussions}

The GCR intensity modulations of solar origin and the diffusion of GCRs in the inner heliosphere are an important topic of study in terms of using cosmic ray observation for space weather studies. The high redundancy of the GRAPES-3 muon telescope allowed an almost uninterrupted stream of data for the years from 2005-2010, which made it possible to explore the interplanetary magnetic field dependence of GCR. We have effectively removed the atmospheric modulations present in the muon intensity by applying the FFT method. Muon intensity data obtained after the pressure and temperature correction was in anti-correlation with the interplanetary magnetic field. From this we determined the dependence of interplanetary magnetic field (B) on the GCR intensity, which is shown in the figure 3. We have investigated this considering the diffusion of GCRs in the heliosphere, which we considered as a magnetic shell of varying intensity. The GCR flux depends up on the diffusion coefficient and the density gradient, we have used two independent diffusion coefficients and the free parameter to used to match the data was the power factor that determines the dependence of Larmor radius on density gradient. Using the $D_{\perp}$ from Candia \& Roulet (2004) we obtained the power index value $\mathrm{x}=0.3148$ and using $D_{\perp}$ from Snodin et al (2016) we obtained the power index value $x=0.6047$. The difference of the power index from both the approximations states that we need to re investigate the diffusion approximation of GCRs in turbulent magnetic 
field. The dependence of magnetic field on GCR flux obtained from GRAPES-3 can be used as a seed for this investigation. GRAPES-3 tracking muon telescope can look in 169 directions which corresponds to different rigidities, this observational capability can be used to find the rigidity dependence of GCRs flux, which can be calculated using the same instrument unlike using different instruments for different rigidities.

\section{Acknowledgments}

We are grateful to D.B. Arjunan, G.P. Francis, V. Jeyakumar, S. Kingston, K. Manjunath, S. Murugapandian, B. Rajesh, K. Ramadass, C. Ravindran, V. Santoshkumar, M.S. Shareef, C. Shobana, R. Sureshkumar for their role in the efficient running of the experiment. We acknowledge the Goddard Space Flight Center for making the interplanetary magnetic field data available online.

\section{References}

[1] K.P. Arunbabu et al., Astron. Astrophys. 555 (2013) A139.

[2] K.P. Arunbabu et al., Astron. Astrophys. 580 (2015) A41.

[3] P. Subramanian et al., Astron. Astrophys. 494 (2009) 1107.

[4] K. Kudela et al., Space Sci. Rev. 93 (2000) 153.

[5] S.E. Forbush, J. Geophys. Res. 59 (1954) 525.

[6] D. Venkatesan, Badruddin Space Sci. Rev. 52 (1990) 121.

[7] P.K. Mohanty et al., Pramana J. Phys. 81 (2013) 343.

[8] Candia, J., \& Roulet, E., J. Cosmol. Astropart. Phys., 10 (2004), 007

[9] A. P. Snodin et al., MNRAS, 457 (2016), 3975S

[10] S.K. Gupta et al., Nucl. Instrum. Methods A 540 (2005) 311.

[11] T. Nonaka et al., Phys. Rev. D 74 (2006) 052003.

[12] H. Kojima, et al., Astropart. Phys. 62 (2015) 21.

[13] H. Kojima et al., Phys. Rev. D 91 (2015) 121303(R).

[14] S.K. Gupta et al., Nucl. Phys. B Proc. Suppl. 196 (2009) 153.

[15] H. Tanaka et al., J. Phys. G: Nucl. Part. Phys. 39 (2012) 025201.

[16] P.K. Mohanty et al., Proceedings of Science PoS(ICRC2017)357

[17] S.K. Gupta, EPJ Web Conf. 52 (2013) 04005.

[18] P.K. Mohanty, Ph.D. Thesis, TIFR unpublished, (2014).

[19] P.K. Mohanty et al., Astropart. Phys. 79 (2016) 23.

[20] P. Barrett et al., Rev. Mod. Phys. 24 (1952) 133.

[21] K. P. Arunbabu et al., Proceedings of Science PoS(ICRC2017)304

[22] Spangler, S. R., ApJ, 576 (2002), 997 